\title{
Possible cross-cultural role in effectiveness of music therapy in palliative and hospice care
}

\author{
Ozan Bahcivan ${ }^{1}$ (I)
}

Received: 20 February 2017 / Accepted: 16 May 2017 /Published online: 24 May 2017

(C) Springer-Verlag Berlin Heidelberg 2017

To The Editor,

First of all, I would like to congratulate Gallagher and colleagues for their study [1] in which they observed 50 family members to understand family members' perceptions of music therapy experienced by a relative in palliative or hospice care. They concluded that music therapy has a positive impact on the family members of patients and themselves in palliative medicine and hospice settings. However, since this study predominantly focused on the effectiveness and benefit of music therapy in palliative care, music therapy may have detrimental reverse effects in one's quality of life if the music has a different meaning, judgement and concepts to that family member or/and individual. In fact, music does play a significant role in mood change, and the perception of music also differs between individuals cross-culturally [2]. As a result of one culture's, mood judgement can be different than others. Considering that culture plays a significant role in the concepts of effectiveness of music therapy, authors did not specify the ethnic or cultural background of the participants in their study. Therefore, it is important to identify the cultural understanding and the relevant barriers as well as personal norms and values towards music. As a result, stereotyping or a "onesize-fits-all" approach should be avoided in both research and practice. Instead, a client-centred approach should be adopted when using any therapeutic techniques as part of an effective approach in palliative or hospice care. In conclusion, since this study used a self-measurement tool, a question about cultural and ethnic background could be included. Therefore, it would provide a more valid outcome in assessing the impact of music therapy on the family members of patients and themselves in palliative and hospice settings.

\section{Compliance with ethical standards}

Conflict of interest The author declares that he has no conflict of interest.

\section{References}

1. Gallagher LM, Lagman R, Bates D et al (2017 Jan 09) Perceptions of family members of palliative medicine and hospice patients who experienced music therapy. Support Care Cancer. doi:10.1007/ s00520-017-3578-y

2. Lee JH., \& Hu, X. Cross-cultural similarities and differences in music mood perception. In iConference. 2014 Proceedings. doi:10. 9776/14081
Ozan Bahcivan

bahcivanozan@yahoo.com.au

1 Family Haven/SA Pregnancy \& Parenting Counselling Services, Prospect, Adelaide 5082, Australia 